\title{
Expression of CXCR4 and CXCL12 (SDF-1) in Human Prostate Cancers (PCa) In Vivo
}

\author{
Yan-Xi Sun, ${ }^{1}$ Jingcheng Wang, ${ }^{1}$ Charles E. Shelburne, ${ }^{2}$ Dennis E. Lopatin, ${ }^{2}$ Arul M. Chinnaiyan, ${ }^{3}$ \\ Mark A. Rubin, ${ }^{4}$ Kenneth J. Pienta, ${ }^{5}$ and Russell S. Taichman ${ }^{1 *}$ \\ ${ }^{1}$ Department of Periodontics, Prevention, Geriatrics, University of Michigan School of Dentistry, \\ 1011 North University Ave., Ann Arbor, Michigan \\ ${ }^{2}$ Department of Biologic and Materials Sciences, University of Michigan School of Dentistry, \\ 1011 North University Ave., Ann Arbor, Michigan \\ ${ }^{3}$ Department of Pathology and Urology, University of Michigan School of Medicine, \\ 1301 Catherine Road, Ann Arbor, Michigan \\ ${ }^{4}$ Department of Pathology, Brigham and Women's Hospital, 75 Francis Street, Boston, Massachusetts \\ ${ }^{5}$ Division of Hematology and Medical Oncology, Department of Internal Medicine, \\ University of Michigan School of Medicine, 1500 E. Medical Center Drive, Ann Arbor, Michigan
}

\begin{abstract}
Human prostate cancers ( $\mathrm{PCa}$ ) express great variability in their ability to metastasize to bone. The identification of molecules associated with aggressive phenotypes will help to define PCa subsets and will ultimately lead to better treatment strategies. The chemokine stromal-derived factor-1 (SDF-1 or CXCL12) and its receptor CXCR4 are now known to modulate the migration and survival of an increasing array of normal and malignant cell types including breast, pancreatic cancers, glioblastomas, and others. The present investigation extends our previous investigations by determining the expression of CXCR4 and CXCL12 in humans using high-density tissue microarrays constructed from clinical samples obtained from a cohort of over 600 patients. These data demonstrate that CXCR4 protein expression is significantly elevated in localized and metastastic cancers. At the RNA level, human PCa tumors also express CXCR4 and message, but overall, they were not significantly different suggesting post-transcriptional regulation of the receptor plays a major role in regulating protein expression. Similar observations were made for CXCL12 message, but in this case more CXCL12 message was expressed by metastastic lesions as compared to normal tissues. PCa cell lines also express CXCL12 mRNA, and regulate mRNA expression in response to CXCL12 and secrete biologically active protein. Furthermore, neutralizing antibody to CXCL12 decreased the proliferation of bone homing LNCaP C4-2B and PC3 metastastic tumor cells. These investigations provide important new information pertaining to the molecular basis of how tumors may 'home' to bone, and the mechanisms that may account for their growth in selected end organs. J. Cell. Biochem. 89: 462-473, 2003. (c) 2003 Wiley-Liss, Inc.
\end{abstract}

Key words: chemokines; bone; marrow; tumor; osteoblast; metastasis

Prostate cancer $(\mathrm{PCa})$ is a common neoplasm and the second leading cause of cancer deaths in American males [Pienta and Esper, 1993;

Grant sponsor: Tissue Procurement Core of the University of Michigan Comprehensive Cancer Center; Grant number: CA46952; Grant sponsor: University of Michigan Comprehensive Cancer Center Histology and Immunoperoxidase Core; Grant sponsor: Specialized Program of Research Excellence in Prostate Cancer (Developmental Grants); Grant sponsor: University of Michigan Core Centers for Musculoskeletal Disorders; Grant number: AR46024; Grant sponsor: National Institute of health; Grant numbers: DE13701, DE11117; Grant sponsor: Department of Defense; Grant number: DAMD17-02-1-0100.
Parker et al., 1996; Weingartner et al., 1998]. Despite numerous advances, the high mortality rate is principally due to the spread of malignant

Mark A. Rubin's present address is Department of Pathology, Brigham and Women's Hospital, 75 Francis Street Boston, MA.

*Correspondence to: Russell S. Taichman, DMD, DMSc, Department of Periodontics, Prevention, Geriatrics, University of Michigan School of Dentistry, 1011 North University Ave., Ann Arbor, Michigan 48109-1078.

E-mail: rtaich@umich.edu

Received 4 December 2002; Accepted 20 February 2003

DOI 10.1002/jcb.10522 
cells to many tissues including bone [Jacobs, 1983; Koutsilieris, 1993]. As a result, there is a growing interest in the early detection and screening of men for $\mathrm{PCa}$, and for a greater understanding of the mechanisms that lead to metastasis (Met) particularly to bone.

Met is a complicated, multi-step process [Fidler, 1999]. Malignant cells must initially "escape" from the primary tumor, invade into the surrounding tissues and enter into the vascular circulation. If they are able to survive in the blood stream, they must arrest at a secondary target site, cross the vascular barrier, and migrate into the extra vascular connective tissues. Subsequently, the tumor cells must proliferate thereby establishing a secondary (metastatic) colony. These events involve numerous cell-cell and cell-matrix adhesive interactions, which are mediated by cell surface adhesion molecules. Numerous hypotheses have been put forth to explain the predilection of PCa for bone. These include the anatomic configuration of venous drainage system from the prostate to the spine, the presence of "leaky" sinusoids in the bone marrow, the elaboration of "tumorattracting" chemotactic factors by bone and/ or other marrow cells, and the production of growth factors in the marrow that stimulate proliferation and survival of seeded cells [Geldof, 1997a]. All of these possibilities are likely to be important determinants of prostate Met to bone [Geldof, 1997b]. Yet despite our increasing understanding in each of these areas the molecular basis for how these tumors preferentially 'home' to bone remains to be determined.

The Met of circulating prostate carcinoma cells is functionally akin to the homing behavior of hematopoietic cells to the bone marrow where many molecules are known to regulate hematopoietic stem cell homing, participating as both chemoattractants and regulators of cell maturation. Considerable attention has been focused on a large family of chemotactic proteins called chemokines [Baggiolini, 1998]. Chemokines are classified based upon the relative position of cysteine residues near the amino-terminus into four major families: CC, CXC, C, $\mathrm{CX}_{3} \mathrm{C}$. Since the first chemokine receptor (IL-8R) was cloned, more than 20 receptors have been identified. All are members of the large family of seventransmembrane $\mathrm{G}$ protein-coupled receptors with considerable overlap in ligand-receptor specificity, thereby adding considerable complexity to the system.
Chemokines play significant roles in normal development, inflammation, infection, atherosclerotic progression, and angiogenesis [Rottman, 1999; Rossi and Zlotnik, 2000; Gerard and Rollins, 2001]. In addition, they are known to participate in hematopoietic stem cell homing. Here a CXC chemokine stromal-derived factor1 (SDF-1 or CXCL12) and its receptor, CXCR4 appear to be critical determinants in these events [Aiuti et al., 1999b; Kim and Broxmeyer, 1999]. This was demonstrated most convincingly in gene knockout investigations showing that normal fetal liver hematopoiesis occurs in homozygous animals, but marrow engraftment by hematopoietic cells does not [Nagasawa et al., 1996; Aiuti et al., 1999a; Peled et al., 1999]. In addition, CXCR4 expression levels correlate with the ability of human progenitors to engraft into the marrow in nude mice, and antibody to CXCR4 prevented the engraftment of progenitors in the bone marrow [Peled et al., 1999]. Finally, osteoblasts and marrow endothelial cells express CXCL12, which may thereby localize hematopoietic progenitor cells in the marrow [Hamada et al., 1998; Wang et al., 1998; Peled et al., 1999].

Recently, we hypothesized that metastatic prostate carcinomas also utilize the CXCL12/ CXCR4 pathway to localize to the bone marrow [Taichman et al., 2002]. We previously demonstrated that PCa cell lines express CXCR4 and that they adhere to endothelium and migrate in response to CXCL12 [Taichman et al., 2002]. The present investigation further explored this hypothesis by determining whether CXCR4 and CXCL12 are expressed by human $\mathrm{PCa}$ in vivo and if receptor expression alters $\mathrm{PCa}$ cell activities that are consistent with metastatic behavior. These investigations provide important new information pertaining to the molecular basis of how tumors 'home' to bone, and will facilitate the development of new strategies for preventing or minimizing prostate Met.

\section{MATERIALS AND METHODS}

\section{Tissue Microarray}

High-density tissue microarrays were constructed from clinical samples obtained from a cohort of over 600 patients, who underwent radical retro pubic prostatectomy at the University of Michigan as a primary therapy (i.e., no preceding hormonal or radiation therapy) for PCa from 1994 to 1998. These were provided 
from the University of Michigan Comprehensive Cancer Center Histology and Immunoperoxidase Core courtesy of Dr. Mark Rubin (Department of Urology and Internal Medicine, University of Michigan, Ann Arbor, MI) as detail previously [Rubin et al., 2000]. Tumors were graded using the Gleason grading system and examined to identify areas of benign prostate, $\mathrm{PCa}$, and bone Met. The formalin-fixed, paraffin-embedded tissues were dewaxed and placed in a pressure cooker containing $0.01 \mathrm{M}$ buffered sodium citrate solution ( $\mathrm{pH}$ 6.0), boiled and chilled to room temperature for antigen retrieval. The slides were then incubated overnight at room temperature with anti-human CXCR4 antibody diluted 1:100 (10 $\mathrm{mg} / \mathrm{ml})$, MBA171 (IgG2a) (Clone 44708.111, R\&D Systems, Minneapolis, MN). A streptavidin/biotin detection method with 3,3'-diaminobenzidine tetrahydrochloride (DAB) was employed for signal detection and Harris hematoxylin was used as a counter-stain. Immunostaining intensity was scored by a genito-urinary pathologist (MAR) as absent (1), weak (2), moderate (3), or strong (4). Scoring was performed blind using a telepathology system without knowledge of overall Gleason score (e.g., tumor grade), tumor size, or clinical outcome [Perrone et al., 2000].

\section{Secondary Data Analysis for CXCL12 and CXCR4 Expression in Human PCa}

Secondary data analysis was performed on data published by Dhanasekaran et al. [2001]. Using a 10,000 human cDNA micoarray which included $\sim 5,520$ known, named genes and 4,464 ESTs, the authors determined the gene-expression profiles of RNA obtained from more than 50 normal and neoplastic prostate specimens [Dhanasekaran et al., 2001]. Primary data analysis was performed with a Genepix software package on Cy3-to-Cy5 ratios determined for the individual genes along with various other quality-control parameters (e.g., intensity over local background) [Dhanasekaran et al., 2001]. Supplementary information from Dhanasekaran et al. [2001] were imported into a Microsoft Excel database and data clustered into normal associated tissues (NAP), benign hyperplasia (BPH), localized cancer (PCa), and Met. The normalized mean and standard deviation (SD) values for CXCR4 and CXCL12 were evaluated for significance with Instat 4.0 (GraphPAD software) using one-way analysis of variance (ANOVA), with the level of significance set at $P<0.05$.

\section{PCa Cell Lines}

PC-3 cells originally isolated from a vertebral Met of a human PCa patient, were obtained from American Type Culture Collection (Rockville, MD) and maintained in RPMI 1640 containing $10 \%$ fetal bovine serum (FBS), $100 \mathrm{U} / \mathrm{ml}$ penicillin, and $100 \mathrm{mg} / \mathrm{ml}$ streptomycin (hereafter called growth medium). LNCaP cells were isolated from a lymph node of a patient with disseminated bony and lymph node involvement. The LNCaP cells and LNCaP sub lines (C4 and C4-2B cells) were originally obtained from UroCor, Inc. (Oklahoma City, OK). The $\mathrm{VCaP}$ cell line was originally harvested at autopsy from a metastatic lesion to a lumbar vertebral body of a patient with hormone refractory $\mathrm{PCa}$ and aseptically xenografted into SCID mice [Korenchuk et al., 2001]. PCa cell lines were passaged and allowed to grow to confluence over 5 days. The cells were cultured in RPMI Medium 1640 supplemented with 10\% FBS, 1\% penicillin-streptomycin, and 1\% L-glutamine. For the production of $\mathrm{PCa}$ cell conditioned medium, the cells were replated at $2 \times 10^{4}$ cells $/ \mathrm{cm}^{2}$ into 24 -well tissue culture plates and incubated in growth medium. After confluence the cells were washed with phosphate-buffered saline (PBS) and medium replaced with growth medium and then incubated for an additional $72 \mathrm{~h}$. Thereafter, the conditioned media were collected and frozen after passage through a $0.22 \mu \mathrm{M}$ filter. Non-conditioned media were similarly treated and used as a negative control.

\section{Mixed Human Bone Marrow Stromal Cell (BMSC) Conditioned Medium and Human Osteoblasts (HOB)}

Human bone marrow cells were obtained from healthy adult volunteers by iliac crest puncture and aspiration into preservative-free heparin under a protocol approved by the University of Michigan's Investigational Review Board. Mononuclear cells were isolated by density separation on Ficoll-Hypaque (specific gravity 1.077). Following plastic adherence at $37^{\circ} \mathrm{C}$ for $2 \mathrm{~h}$, the non-adherent cells were removed and the cultures were allowed to reach confluence (1-2 weeks). The cells were used after 2nd passage in Iscove's Modified Dulbecco's Medium (IMDM medium; Life Technologies, Grand Island, NY) with 10\% FBS (Life Technologies), $10 \%$ equine serum (Life Technologies) 
and $1 \mu \mathrm{M}$ hydrocortisone (Life Technologies). Conditioned medium was collected from when 2nd passage cells had reached $\sim 85 \%$ confluent. The cells were washed, replaced with serum reduced AIM V medium, incubated for an additional $24 \mathrm{~h}$, then conditioned, and media not exposed to cells were collected and frozen after passage through a $0.22 \mu \mathrm{M}$ filter.

Enriched human osteoblast cultures were established using modifications of methods described by Robey and Termine [1985]. Normal human trabecular bone obtained from patients undergoing orthopedic surgery in accordance with the University of Michigan's Investigational Review Board, was cleaned of loosely adherent tissue, ground to produce a uniform particle size (size $\leq 1 \mathrm{~mm}^{2}$ ) (BioComp Minimill, W. Lorenz, Jacksonville, FL) and incubated in $1 \mathrm{mg} / \mathrm{ml}$ bacterial collagenase (Type B, Boehringer Mannheim Biologicals, Indianapolis, IN) in DMEM. The explants cultured until confluent in a 1:1 (vol/vol) mixture of Ham's F12/ DMEM (Biofluids, Rockville, MD) with low $\mathrm{Ca}^{+2}$ and $10 \%$ heat inactivated FBS. Thereafter, the cultures were maintained in calcium replete Ham's F12/DMEM (1:1 vol/vol) medium containing $10 \%$ heat inactivated serum, antibiotics, $10 \mathrm{mM} \beta$-glycerol phosphate, and $10 \mu \mathrm{g} /$ $\mathrm{ml}$ L-ascorbate. Characterization and culture of the resulting cells has been described in detail previously [Taichman and Emerson, 1994; Taichman and Emerson, 1998].

\section{CXCL12 ELISA}

Conditioned medium was collected over time and stored at $-80^{\circ} \mathrm{C}$ until assayed by antibody sandwich ELISA assembled with components purchased from R\&D Systems (sensitivity 31.25 $\mathrm{pg} / \mathrm{ml}$, range $62.5-5,000 \mathrm{pg} / \mathrm{ml}$; R\&D Systems).

\section{Proliferation Assays}

PCa cells were washed three times in PBS, and $5 \times 10^{3}$ cells plated in triplicate in 96-well flat-bottomed tissue culture plates (BD Biosciences, Palo Alto, CA) in $0.1 \mathrm{ml}$ in growth medium. Log serial dilutions of recombinant human CXCL12 was added alone or in combination with BMSC conditioned medium $(0,10$, $25 \%)$ over a dose range of $(0.1-200 \mathrm{ng} / \mathrm{ml})(\mathrm{R} \& \mathrm{D}$ Systems). Thereafter, the cultures were incubated in an atmosphere of $5 \% \mathrm{CO}_{2}$ and $5 \% \mathrm{O}_{2}$ at $37^{\circ} \mathrm{C}$ for 4 days. Proliferation was quantified by either colorimetric assay using 3-(4,5dimethylazol-2yl)-2,5-diphenyltetrazolium bro- mide (MTT) or sodium 3'-(1-(phenylaminocarbonyl)-tetrazolium)-bis (4-methoxy-6-nitro(XTT) (Sigma, St. Louis, Missouri) and read on a multiwell scanning spectrophotometer (Molecular Devices Corp., Sunnyvale, CA). The effect of PCa-derived CXCL12 proliferation was evaluated by the addition of neutralizing antiCXCL12 monoclonal antibodies (R\&D Systems) or IgG control, or vehicle were added to a final concentration of $120 \mu \mathrm{g} / \mathrm{ml}$ daily for 3 days, where proliferation was determined by manual hemocytometer counting or quantified by MTT assay.

\section{Real Time RT-PCR}

The PCa cell lines (LNCaP, LNCaP C4-2B, and PC3) were passaged and cultured in RPMI Medium 1640, supplemented with $10 \%$ FBS, $1 \%$ penicillin-streptomycin, and 1\% L-glutamine. After confluence, the media were removed and replaced with serum free media, and CXCL12 at 0 or $200 \mathrm{ng} / \mathrm{ml}$, were added for $1-3 \mathrm{~h}$. The cells were then lysed directly in Trizol ${ }^{\circledR}$ according to the directions of the manufacturer (Trizol, Invitrogen, Carlsbad, CA). RNA integrity and purity was checked by electrophoresis with ethidium bromide and absorbance at $\mathrm{A}_{260} / \mathrm{A}_{280}$. First strand cDNA synthesis was performed on $1 \mu \mathrm{g}$ of total RNA using random hexamers and QuantiTect SYBR $^{\mathrm{R}}$-Green PCR Kit for quantitative, two step real-time RT-PCR (Qiagen, Valencia, CA). Amplification primers were designed using PrimerExpress ${ }^{\mathrm{TM}}$ software (Applied Biosystems, Foster City, CA) to cross intron/exon boundaries and were validated by (i) sequencing the resulting product using the University of Michigan DNA Sequencing Core Facilities, and by (ii) determining cross-reactivity using BLAST (http://www.ncbi. nlm.nih.gov/BLAST/), those with the lowest potential were synthesized. The sequences of the forward and reverse primers of CXCR4 were $5^{\prime}$-TGACGGACAAGTACAGGCTGC and 5'-CCAGAAGGGAAGCGTGATGA, respectively. The sequences of the forward and reverse primers of CXCL12 were 5'-TGCCAGAGCCAACGTCAAG, 5'-CAGCCGGGCTACAATCTGAA. The PCR product was detected as an increase in fluorescence with the ABI PRISM 7700 instrument (Applied Biosystems). PCR was performed with $12.5 \mu \mathrm{l}$ of SYBR PCR master mixture, each of the primers at a concentration of $100 \mathrm{nM}$, and $1 \mu \mathrm{l}$ of RT product in a total volume of $25 \mu \mathrm{l}$. The 2 -step $\mathrm{PCR}$ reaction $\left(95^{\circ} \mathrm{C}\right.$ 
for $15 \mathrm{~s}, 60^{\circ} \mathrm{C}$ for $60 \mathrm{~s}$ ) was run for 40 cycles after an initial single cycle of $95^{\circ} \mathrm{C}$ for $15 \mathrm{~min}$ to activate the Taq polymerase. The mRNA levels were expressed either in absolute copy number or relative copy numbers (\% control) normalized against 18S mRNA (Ambion QuantumRNA ${ }^{\mathrm{TM}}$ 18S Universal Primers) and a standard curve constructed from serial dilutions of a purified CXCL12 and CXCR4 cDNA fragment cloned from PCR product obtained by classical PCR.

\section{Statistical Analysis}

Differences in CXCR4 protein expression were evaluated statistically using the mean scores from each case for each prostate tissue type (e.g., benign, localized $\mathrm{PCa}$, and hormonerefractory metastatic $\mathrm{PCa}$ ). To test for significant differences in the mean CXCR4 protein expression among all tissue types, we performed a one-way ANOVA test. To determine differences between all pairs (e.g., localized PCa vs. benign), we performed a post-hoc analysis using the Scheffe method [Rubin et al., 2000]. The mean expression scores for all benign, localized $\mathrm{PCa}$, and bone Met examined cases are presented in a graphical format using error bars with $95 \%$ confidence intervals (CI). Culture data were expressed as mean \pm SD. Statistical differences between the means for the different groups were evaluated with Instat 4.0 (GraphPAD software) using one-way ANOVA, with the level of significance at $P<0.05$. All in vitro experiments were repeated two to three times with triplicate samples and similar results were obtained.

\section{RESULTS}

Our premise is that CXCL12 and its CXCR4 receptor help to define the bone specific Met of prostate carcinomas. To address the hypothesis, we previously demonstrated that osteoblasts in the bone marrow express CXCL12, and that PCa cell lines express CXCR4 and are functionally responsive to the ligand. In the present

Fig. 1. The expression of CXCR4 in human prostate cancers (PCa). Formalin-fixed paraffin-embedded tissues were dewaxed and placed in a pressure cooker containing $0.01 \mathrm{M}$ buffered

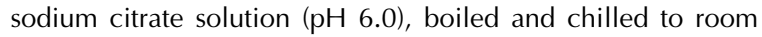
temperature for antigen retrieval. The slides were incubated overnight at room temperature with anti-human CXCR4 antibody diluted 1:100 (10 mg/ml). MBA171 (IgG2a) (Clone 44708.111, R\&D Systems, Minneapolis, MN). A streptavidin/biotin detection method with 3,3'-diaminobenzidine tetrahydrochloride (DAB) investigation, we determined if PCA cells express the CXCR4 receptor in humans.

To examine the widest range of $\mathrm{PCa}$ specimens, high-density tissue microarrays were constructed from clinical samples obtained from a cohort of over 600 patients, who underwent radical retro pubic prostatectomy at the University of Michigan as a primary therapy (i.e., no preceding hormonal or radiation therapy) for clinically localized PCa from 1994 to 1998 with approval from University of Michigan IRB (Provided by Dr. Mark Rubin, University of Michigan from the Rapid Autopsy Program which have been described in detail previously [Rubin et al., 2000]). Tumors were graded using the Gleason grading system and examined to identify areas of benign prostate, $\mathrm{PCa}$ and bone Met. Staining of four tissue microarrays with monoclonal antibody revealed moderate-tostrong CXCR4 protein expression in clinically localized PCa samples with cytoplasmic and nuclear localization (Fig. 1A,B,C). Levels of CXCR4 protein expression in the malignant epithelia were greater than in the benign epithelia (Fig. 1D), whereas normal epithelium predominately demonstrated weak cytoplasmic with strong nuclear staining (Fig. 1D). Both prostatic intraepithelial neoplasia and some atrophic lesions, thought to be potentially precancerous lesions demonstrated cytoplasmic staining of CXCR4 but as there were too few samples they were not included in our analysis [Putzi and De Marzo, 2000]. High-grade (Gleason score, $4+4=8$ ) (Fig. 1E) and lowgrade $\mathrm{PCa}$ (not shown) demonstrated strong cytoplasmic staining. Similar staining was observed in bone metastases (Fig. 1F).

Quantitative analysis of the expression CXCR4 receptors in human PCa was performed by scoring protein expression as negative ( score $=1$ ), weak (2), moderate (3), and strong (4). Differences in CXCR4 protein expression were evaluated statistically using the mean score results from each case for each prostate tissue type. As is shown in Figure 2, immuno-

was employed for signal detection. Harris hematoxylin was used as a counter-stain CXCR4 is over expressed in PCa. Representative elements of a tissue microarray stained with anti-CXCR4 antibody showing localized and invasive prostate carcinomas (A, B, C). Staining is weak in benign prostatic hyperplasia (BPH) (D), but strong in the cytoplasm of localized PCa (E), and metastastic bone lesions $(\mathbf{F})$. Original magnification $\times 1(\mathrm{~A}), \times 10(\mathrm{~B}), \times 20(\mathrm{C})$, $\times 200(\mathrm{D}, \mathrm{E}, \mathrm{F})$. [Color figure can be viewed in the online issue, which is available at www.interscience.wiley.com.] 
A
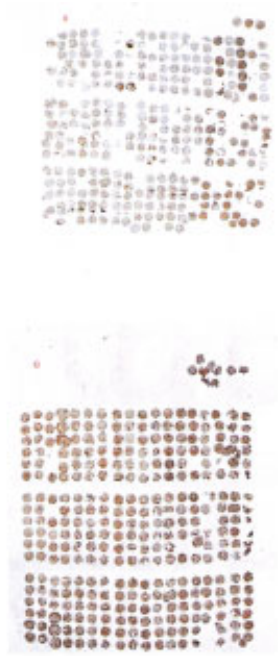

B

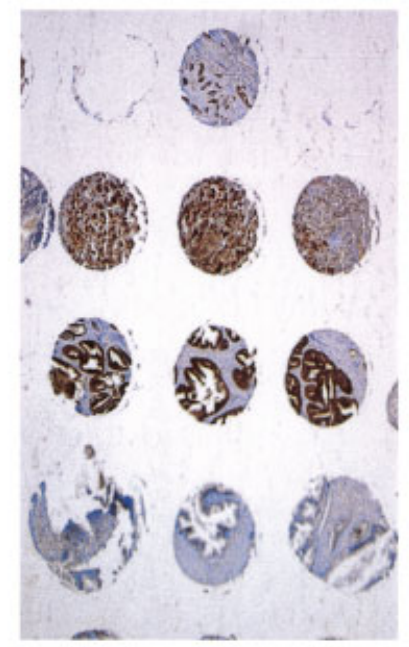

C
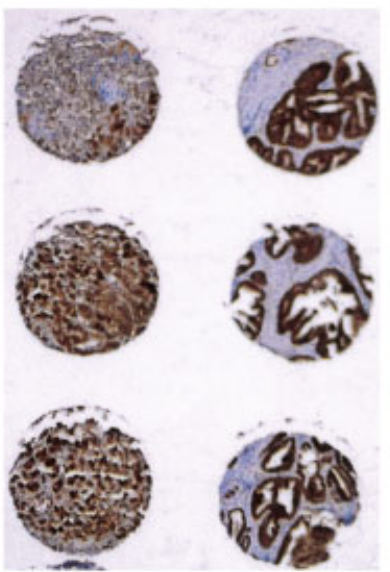

D

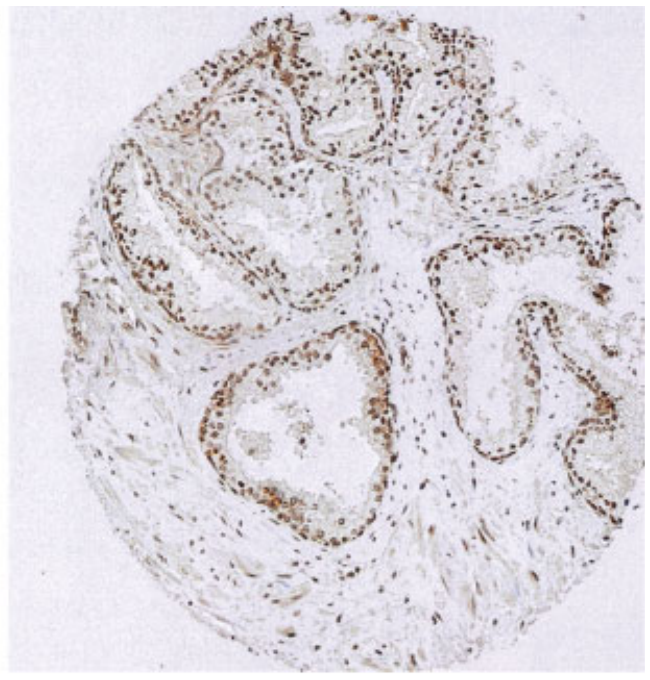

E

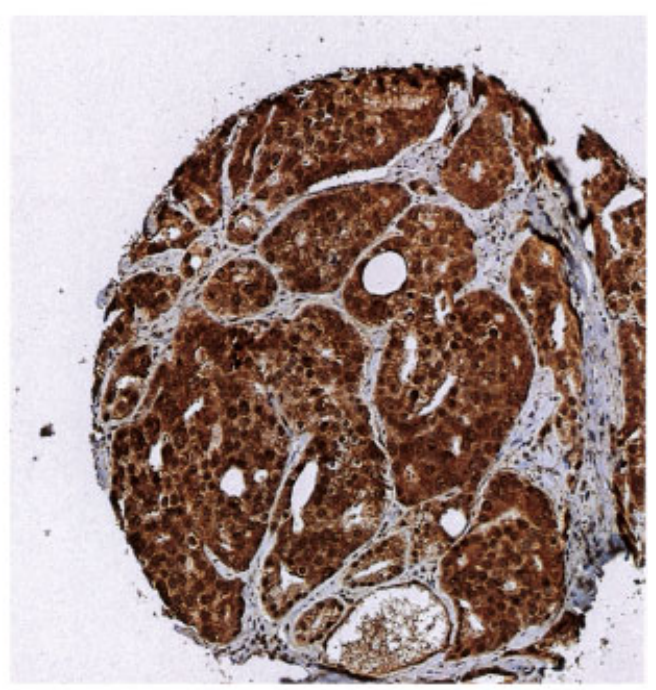

F

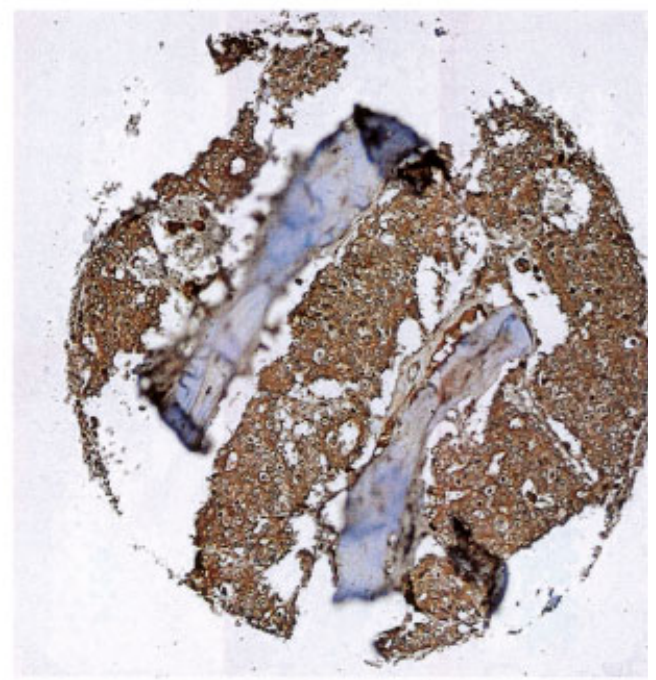

Fig. 1. 


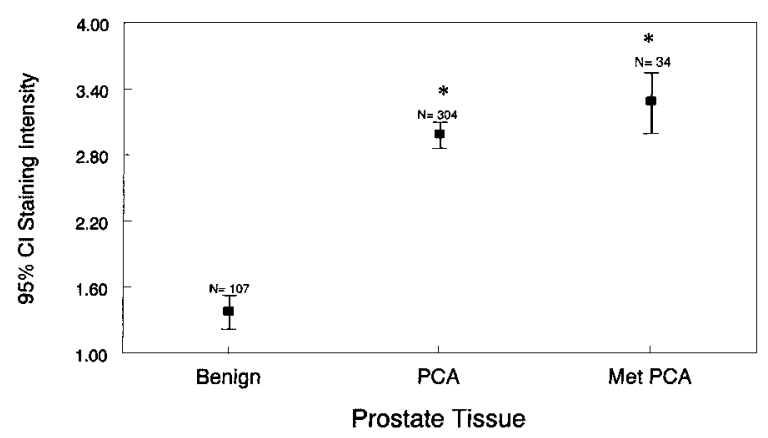

Fig. 2. Quantitative evaluation of the expression of CXCR4 is in $\mathrm{PCa}$ and bone metastases. Immunostaining intensity was scored as either absent (1), weak (2), moderate (3), or strong (4). The mean expression scores for all benign, localized PCa, and bone metastasis (Met) examined cases are presented in a graphical format using error bars with 95\% confidence intervals (CI). Statistically significant differences were noted between benign hyperplasia (BPH) and localize cancer (PCa) or metastastic cancer (Met PCA) for $P<0.05(*)$.

histochemical data show that the expression of CXCR4 increased with increasing tumor aggressiveness. Secondary data analysis of microarray data collected for the purposes of a prior investigation was also performed to determine CXCR4 mRNA levels [Shibuta, 2002]. Using computed $t$-statistics, we observed that the CXCR4 RNA levels in benign hyperplastic, localized and metastatic tumors were elevated relative to normal adjacent tissues (Fig. 3). In this case, however, the mean peak in CXCR4 mRNA expression was observed in the benign hyperplastic disease with CXCR4 mRNA expression declining with increasing tumor grade, although statistically there were no significant differences.

During organogenesis, CXCR4 and CXCL12 are known to have dynamic and complementary expression patterns [McGrath et al., 1999]. These include the apposed expression of CXCR4 and CXCL12 in mesoderm/ectoderm during gastrulation, vascular endothelium/mesoderm, thyroid endodermal epithelium/mesenchyme mesenchyme [McGrath et al., 1999]. We, therefore, examined whether CXCL12 was expressed by human PCa themselves in a similar fashion. Unfortunately, we were not able to consistently demonstrate CXCL12 at the protein level using our tissue microarrays, although weak nuclear staining for CXCL12 was frequently observed in localized PCa cancers (data not presented). Therefore, CXCL12 expression was examined at the level of mRNA in human PCa by secondary data analysis of microarray data collected

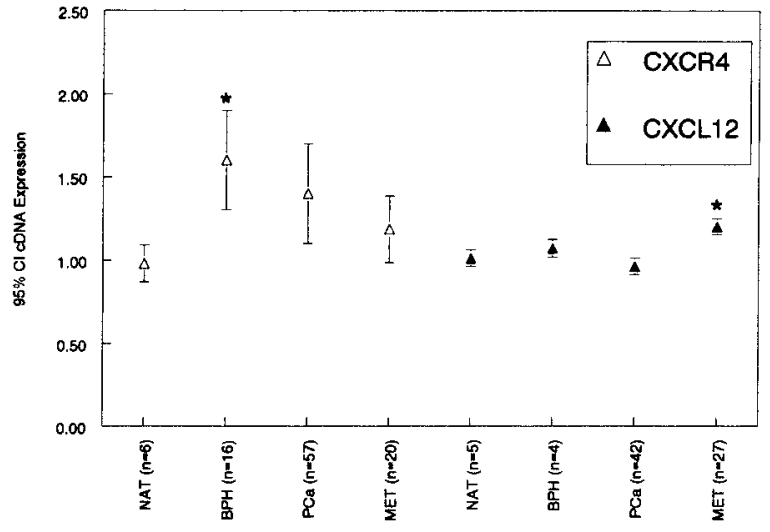

Fig. 3. Expression of CXCR4 and CXCL12 mRNA in human PCa. Secondary data analysis of CXCR4/CXCL12 mRNA was performed using data published by Dhanasekaran et al. [2001]. Primary data analysis was performed with a Genepix software package on Cy3-to-Cy5 ratios determined for the individual genes along with various other quality-control parameters (e.g., intensity over local background) [Dhanasekaran et al., 2001]. Data were clustered into normal associated tissues (NAP), BPH, and localize cancer ( $\mathrm{PCa}$ ) or metastastic cancer (Met PCa). A graphical format using error bars with $95 \% \mathrm{Cl}$ is presented where significance from NAT for CXCL12 and CXCR4 was determined using one-way analysis of variance (ANOVA) with the level of significance set at $P<0.05\left(^{*}\right)$.

for the purposes of a prior study [Shibuta, 2002]. The data demonstrate that metastastic PCa cells express significantly more mRNA for CXCL12 than do normal prostatic tissues (Fig. 3). There was no significant differences observed between normal tissues and $\mathrm{BPH}$ and localized tumors.

Given that the expression of CXCL12 mRNA was elevated in metastastic disease, but not in the localized tumor, we hypothesized that CXCL12 might serve as a growth factor for metastatic disease once the tumor had lodged in a metastastic site. To test this possibility, $\mathrm{PCa}$ cell lines were examined for their expression and secretion of CXCL12 as well as CXCR4. Here real-time RT-PCR was employed to determine the level to which the messages were expressed in PCa cells. In addition, we evaluated whether CXCL12 and CXCR4 mRNA could be regulated by CXCL12. For direct comparisons between the experimental conditions and cell types, a CXCL12 and CXCR4 mRNA standard curve were generated and used to normalize cycle data to copy number (Fig. 4A). The data demonstrate that each of the PCa cells examined expressed mRNA for CXCRL12 as well as CXCR4 (Fig. 4A). The basal levels of CXCL12 mRNA were highest in the LNCaP C42B and LNCaP cell lines followed by the PC3 

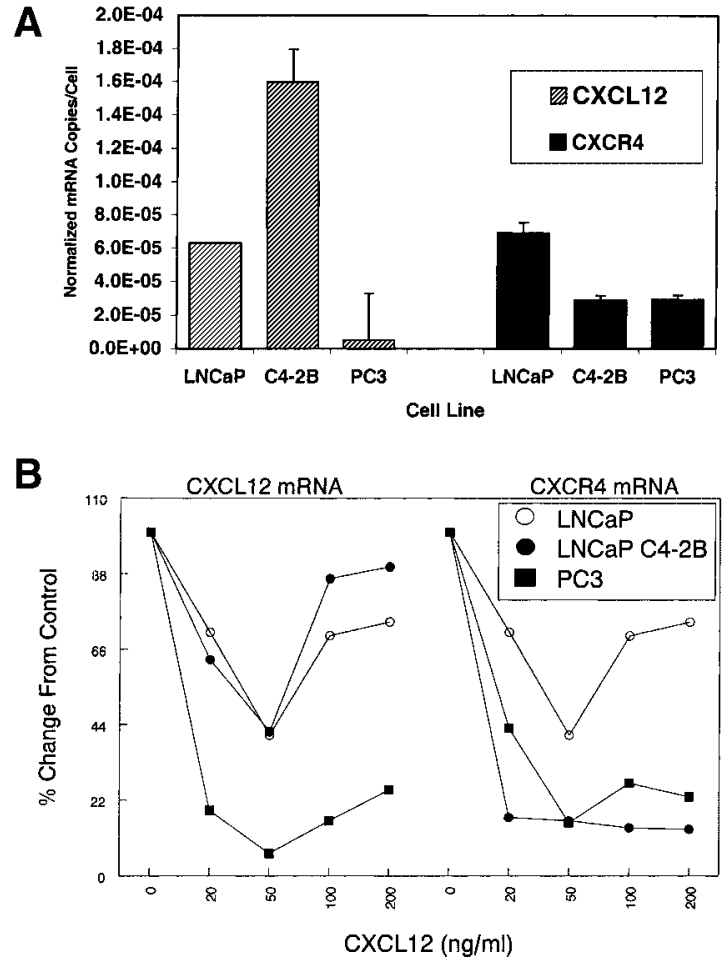

Fig. 4. Real time RT-PCR determination of CXCR4 and CXCL12 expression level. RNA recovered from LNCaP, LNCaP C4-2B, and PC3 cells was reverse transcribed and the levels of CXCR4 and CXCL1 2 mRNA determined by real-time PCR. Absolute copy numbers were obtained from standard curves constructed from serial dilutions of purified CXCL12 and CXCR4 DNA and normalized against $18 \mathrm{~S}$ mRNA.

cells. CXCR4 mRNA levels were highest in the LNCaP cells, with no significant difference in expression between the LNCaP C4-2B and PC3 cell lines (Fig. 4A). When the cells were stimulated for $1 \mathrm{~h}$ with increasing concentrations of recombinant CXCL12, mRNA for CXCL12 and CXCR4 rapidly declined in each cell type demonstrating the functionality of CXCR4 receptors, and that CXCL12 mRNA levels are also influenced by the presence extracellular CXCL12 (Fig. 4B).

Given that all of metastastic cell lines expressed CXCL12 mRNA (Fig. 3) and responded to CXCL12 by altering their expression of CXCR4 and CXCL12 RNA (Fig. 4), PCa cells were next examined to determine if they express CXCL12 protein. For these investigations, protein levels of CXCL12 was determined using an commercial ELISA (R\&D Systems). As demonstrated in Figure 5, each of the cells secreted CXCL12 into their media. While the levels of CXCL12 secreted by PCa were significantly less than those secreted by cultured primary HOB

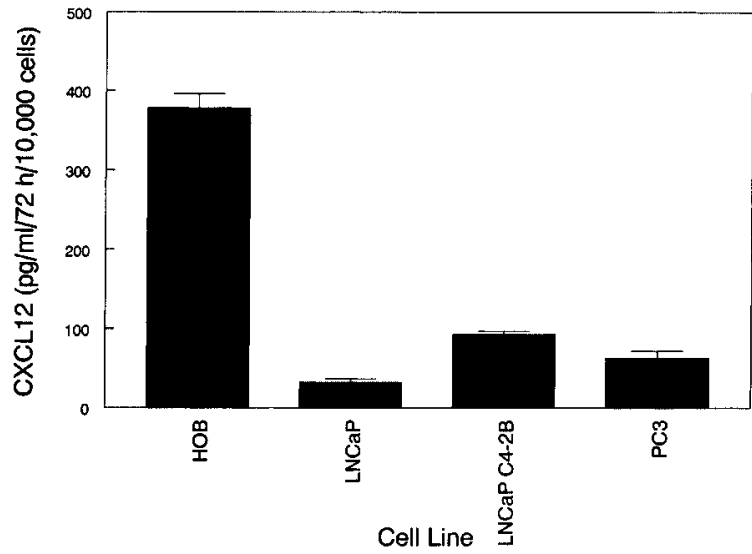

Fig. 5. PCa cell lines secrete CXCL12. Conditioned media from primary human osteoblasts $(\mathrm{HOB})$ and $\mathrm{PCa}$ cell lines were collected at $72 \mathrm{~h}$ and stored at $-80^{\circ} \mathrm{C}$ until assayed by antibody sandwich ELISA assembled with components purchased from R\&D Systems. The data are presented as mean and standard deviation (SD) for triplicate determinations, and demonstrate secretion of CXCL12 by PCa cells in culture.

(Fig. 5), the levels of CXCL12 achieved by the $\mathrm{PCa}$ cells are never-the-less biologically relevant for they are able to synergize with G-CSF to stimulate the proliferation of murine myelomonocytic cells (data not presented).

To determine whether the CXCL12 produced by PCa cells might serve as a growth factor to stimulate proliferation of the PCa cells in an autocrine-like fashion, increasing concentrations of recombinant CXCL12 were add PCa cell cultures under normal growth conditions. After 3 days cell proliferation was evaluated colorimetrically using MTT (Sigma, St. Louis, MO). CXCL12, itself did not alter the growth rate of any of the cell lines examined (data not presented). Similar investigations were then performed under serum free conditions and these too failed to demonstrate a proliferative response of any of the $\mathrm{PCa}$ cells tested to exogenous CXCL12. We next evaluated if conditioned medium derived from BMSCs could synergize with recombinant CXCL12 to stimulated $\mathrm{PCa}$ growth. Our rationale here was that since $\mathrm{PCa}$ cells metastasize to sites of high CXCL12 production including the marrow, perhaps there were factors missing in our cultures that when combined with CXCL12 would stimulate PCa cell growth. These studies were undertaken based upon the reports that CXCL12 alone does not stimulate early hematopoietic progenitor cell growth but is known to synergize with other growth factors [Grafte-Faure et al., 2000; Hodohara et al., 2000]. The combination of 
0,10 , and $25 \%$ human stromal cell conditioned medium to recombinant CXCL12 also failed to stimulate PCa proliferation, although the conditioned medium itself stimulated proliferation (data not presented). Similar observations were made for PCa cell lines grown in the presence of human osteoblast conditioned medium (data not shown).

Finally, we directly addressed if CXCL12 derived from the PCa cells themselves acts in an autocrine fashion to stimulate growth by neutralizing the CXCL12 produced by the PCa cells themselves. For these studies neutralizing antibody to CXCL12 was administered daily under normal serum or serum free conditions. At $72 \mathrm{~h}$, the cells were recovered and counted. Antibody to CXCL12 significantly decreased the number of LNCaP C4-2B, PC3 recovered from the cultures relative to isotype matched or vehicle controls in either normal $(10 \%)$ or serum-free cultures (Fig. 6). Similar treatments of LNCaP, had little or no effect on proliferation. To verify these findings, these investigations were also performed on a novel PCa cell line derived from a vertebral metastatic lesion (VCaP) which was harvested at autopsy fro a metastatic lesion to a lumbar vertebral body of a patient with hormone refractory $\mathrm{PCa}$ [Korenchuk et al., 2001]. Like the other PCa cells, these cells also expressed CXCR4 and CXCL12 (data not presented). Once again antibody to CXCL12 significantly decreased the number of $\mathrm{VCaP}$ recovered from the cultures relative to isotype matched or vehicle controls in either normal (10\%) or serum-free cultures (Fig. 6). Together these data suggest that in addition to utilizing CXCR4/CXCL12 as a metastastic mechanism, as a growth factor for $\mathrm{PCa}$ cells, CXCL12 may be used by to establish metastases in CXCL12 rich organs once they have metastasized.

\section{DISCUSSION}

In the present report, we examined human PCa for their expression of CXCR4 and CXCL12, as they play a major role in hematopoietic stem cell homing to the marrow. Using high-density tissue microarrays constructed from clinical samples from a large cohort of patients, we observed that the expression of CXCR4 correlated with increasing malignancy. The data demonstrate moderate-to-strong CXCR4 protein expression in clinically localized $\mathrm{PCa}$ samples

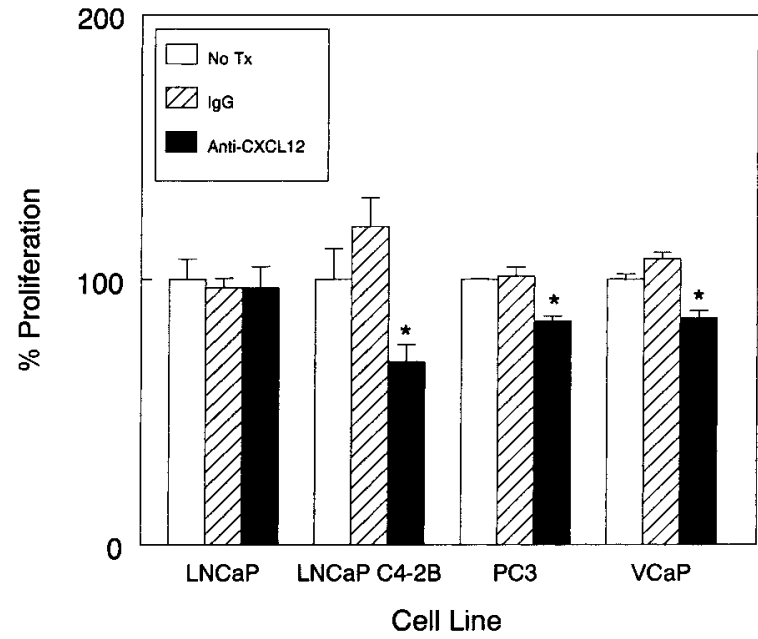

Fig. 6. Neutralizing Antibody to CXCL12 inhibits LNCaPC4-2B cell proliferation. LNCaP C4-2B cells were seeded overnight at 2,000 cells/well in 96 -well plates in medium containing $10 \%$ FCS. Thereafter, the medium was removed and fresh growth medium was replaced with serum reduced medium. Neutralizing murine anti-human neutralizing CXCL12 cytokine antibody (or control) were added daily to a final concentration of $120 \mu \mathrm{g} /$ $\mathrm{ml}$. Absolute cell numbers were determined by manual hemocytometer counting and reported as mean $\pm S D(n=4)$ at 4 days. *Significant difference from antibody or vehicle control, $P<0.05$.

with cytoplasmic and nuclear localization observed where the levels of CXCR4 protein expression in malignant epithelia were highest. Normal epithelium demonstrated predominately weak cytoplasmic staining. Both prostatic intraepithelial neoplasia and some atrophic lesions, also demonstrated cytoplasmic staining of CXCR4, however, due to the small sample sizes, were not included in our data. High-grade (Gleason score, $4+4=8$ ) and low-grade $\mathrm{PCa}$ (not shown) also demonstrated strong cytoplasmic staining. Similar results were observed in boney metastases.

Secondary data analysis using a large cohort study of gene-expression profiles from RNA obtained from more than 50 normal and neoplastic prostate specimen also suggested a relationship between CXCR4 mRNA levels and clinical parameters [Dhanasekaran et al., 2001]. CXCR4 expression was elevated in BPH, localized $\mathrm{PCa}$ and metastatic cancers although no significant differences were seen between the $\mathrm{BPH}$ and increasing tumor grade. This may suggest that CXCR4 expression in PCa may be regulated at the protein level in vivo where rapid turn over of the receptor from intracellular pools has been reported [Cheng et al., 2000]. At present little is known with regard to 
the CXCR4 regulation in tumors, and whether expression levels correlates with clinical outcomes. Far more is known with regards to the role of CXCR4 in other diseased states where CXCR4 serves as a co-receptor for some HIV-2 isolates [Caruz et al., 1998; Gupta and Pillarisetti, 1999]. During development, CXCR4 is expressed in a broad range of tissues, including brain, lymph node, and small intestine [Wegner et al., 1998; McGrath et al., 1999] functioning in a number of normal processes including visualization by regulating endothelial branching and remodeling and CNS development [Feil and Augustin, 1998; Lazarini et al., 2000]. CXCR4 is highly expressed on monocytes, B cells, and naive $\mathrm{T}$ cells in peripheral blood as well as early hematopoietic progenitor cells [Aiuti et al., 1999b]. In fact, CXCR4 expression is elevated in $\mathrm{CD} 34^{+}$progenitors in bone marrow relative to $\mathrm{CD} 34^{+}$progenitors in the peripheral blood [Aiuti et al., 1997]. Suggesting that differential expression of CXCR4 may be involved in maintaining hematopoietic progenitor cells in the marrow and regulating stem cell trafficking [Aiuti et al., 1997].

Most germane to the present report are data that demonstrate that tumor progression may be associated with alterations in CXCR4 mRNA levels. Muller et al. [2001] reported that CXCR4 and CXCL12 are central players in regulating Met by demonstrating that normal breast tissues express little CXCR4, whereas breast neoplasms express high levels of CXCR4. In this report, antibody to CXCR4 blocked the metastatic spread of the tumors to the lung and lymph nodes [Muller et al., 2001]. Similarly, CXCR4 mRNA levels are elevated in glioblastoma multiforme in regions of angiogenesis and degeneration, but deceased in areas of rapid cell proliferation and may be a general characteristic of neuroblastoma cells that supports their preferential Met into the bone marrow [Geminder et al., 2001]. Results consistent with these have also been reported for human melanoma cell lines and melanoma cells that had macroscopically infiltrated draining lymph nodes [Robledo et al., 2001], and for pancreatic and renal carcinomas [Koshiba et al., 2000; Gerritsen et al., 2002]. These results are also consistent with those of Zeelenberg et al. who transfected CXCL12 fused to a KDEL sequence into mouse T cell hybridoma TAM2D2. The SDF-KDEL fusion protein was retained in the endoplasmic reticulum by the KDEL- receptor, and bound to CXCR4, which was, therefore, also retained. This prevented the Met of the cell line to many different tissues [Zeelenberg et al., 2001]. Yet CXCR4 is not a generalized feature of all carcinomas. Several digestive tract cancers including colon, esophageal, gastric, and premalignant liver disease (hepatitis $\mathrm{C}$ viral infected livers) fail to alter their expression of the receptor. Moreover, CXCR4 expression is even reduced in hepatocellular carcinomas [Mitra et al., 1999]. These investigations suggest that CXCR4 may play an important role in neoplastic events including the Met of a wide variety of solid tumors including prostate carcinomas [Muller et al., 2001].

An other intriguing aspect of this investigation is the observation that both members of the CXCR4/CXCL12 chemokine axis are expressed by $\mathrm{PCa}$ cells in vitro, and in in situ tumors express CXCL12 mRNA as well as CXCR4. From these observations found that $\mathrm{PCa}$ cell proliferation was influenced by CXCL12. This observation is quite surprising given the possibility that $\mathrm{PCa}$ may utilize the CXCR4 to localize into end organs that supply high levels of ligand (e.g., bone, lung, liver, lymphnode [Muller et al., 2001]). Moreover these observations are consistent with our previous demonstration that binding of CXCL12 to CXCR4 in PCa cells activate ERK signaling that frequently stimulates proliferation [Taichman et al., 2002]. Recently Zhou et al. [2002] demonstrated that over expression of CXCR4 in glioblastoma cell lines enhanced their growth in soft agar. As with our findings the treatment of the glioblastoma cell lines with antibody to CXCR4, or CXCL12, inhibited proliferation suggesting that $C X C R 4$ gene is required possibly by preventing apoptosis upon serum withdrawn [Zhou et al., 2002]. Of note, not all tumors expressing CXCR4 proliferate in response to CXCL12 including rhabdomyosarcoma cells [Libura, 2002]. Thus CXCL12induced proliferation, may represent an additional mechanism for survival utilized by malignant cells in those PCa cells that metastasize to bone. Moreover, perhaps the ability to respond in an autocrine or paracrine fashion to CXCL12 provides a clue as to why some tumors grow well in CXCL12 rich environments (e.g., bone) while others do not [Guise, 2002].

In summary, $\mathrm{PCa}$ present great variability in their ability to metastasize to bone. The identification of molecules associated with the 
aggressive phenotypes will define PCa subsets that will ultimately lead to better treatment strategies. The CXCL12/CXCR4 axis is now known to modulate the migration and survival of an increasing array of normal and malignant cell types (including breast [Moore, 2001; Muller et al., 2001], pancreatic cancers [Koshiba et al., 2000] glioblastomas [Sehgal et al., 1998; Rempel et al., 2000], and others [Shibuta et al., 1997; Begum et al., 1999; Mitra et al., 1999; Koshiba et al., 2000; Geminder et al., 2001; Robledo et al., 2001]). Our data is consistent with the emerging concept that CXCL12 and CXCR4 expression are associated with increasing malignant potential. The mechanisms of exactly how CXCL12/CXCR4 participate and direct the invasive patterns of $\mathrm{PCa}$ cells remains to be determined. Future studies will be required to characterize the mechanisms whereby CXCL12 alters the adhesion to bone marrow endothelial cells and invasion into the extracellular matrix and to directly demonstrate that blockade of this axis inhibits skeletal metastases.

\section{ACKNOWLEDGMENTS}

We are indebted to Dr. Abraham Schneider, Dr. Laurie McCauley, and Dr. Nadir Osman for their insightful discussions.

\section{REFERENCES}

Aiuti A, Webb IJ, Bleul C, Springer T, Gutierrez-Ramos JC. 1997. The chemokine SDF-1 is a chemoattractant for human CD34+ hematopoietic progenitor cells and provides a new mechanism to explain the mobilization of CD34+ progenitors to peripheral blood. J Exp Med 185:111-120.

Aiuti A, Tavian M, Cipponi A, Ficara F, Zappone E, Hoxie J, Peault B, Bordignon C. 1999a. Expression of CXCR4, the receptor for stromal cell-derived factor-1 on fetal and adult human lympho-hematopoietic progenitors. Eur J Immunol 29:1823-1831.

Aiuti A, Tavian M, Cipponi A, Ficara F, Zappone E, Hoxie J, Peault B, Bordignon C. 1999b. Expression of CXCR4, the receptor for stromal cell-derived factor-1 on fetal and adult human lympho-hematopoietic progenitors. Eur J Immunol 29:1823-1831.

Baggiolini M. 1998. Chemokines and leukocyte traffic. Nature 392:565-568.

Begum NA, Shibuta K, Mori M, Barnard GF. 1999. Reduced expression of the CXCR4 receptor mRNA in hepatocellular carcinoma and lack of inducibility of its ligand alpha-chemokine hIRH/SDF1alpha/PBSF in vitro. Int J Oncol 14:927-934.

Caruz A, Samsom M, Alonso JM, Alcami J, Baleux F, Virelizier JL, Parmentier M, Arenzana-Seisdedos F. 1998. Genomic organization and promoter characterization of human CXCR4 gene. FEBS Lett 426:271-278.
Cheng ZJ, Zhao J, Sun Y, Hu W, Wu YL, Cen B, Wu GX, Pei G. 2000. Beta-arrestin differentially regulates the chemokine receptor CXCR4-mediated signaling and receptor internalization, and this implicates multiple interaction sites between beta-arrestin and CXCR4. J Biol Chem 275:2479-2485.

Dhanasekaran SM, Barrette TR, Ghosh D, Shah R, Varambally S, Kurachi K, Pienta KJ, Rubin MA, Chinnaiyan AM. 2001. Delineation of prognostic biomarkers in prostate cancer. Nature 412:822-826.

Feil C, Augustin HG. 1998. Endothelial cells differentially express functional CXC-chemokine receptor-4 (CXCR4/fusion) under the control of autocrine activity and exogenous cytokines. Biochem Biophys Res Commun 247: 38-45.

Fidler IJ. 1999. Critical determinants of cancer metastasis: Rationale for therapy. Cancer Chemother Pharmacol 43:S3-S10.

Geldof AA. 1997a. Models for cancer skeletal metastasis: A reappraisal of Batson's plexus. Anticancer Res 17: 1535-1539.

Geldof AA. 1997b. Models for cancer skeletal metastasis: A reappraisal of Batson's plexus. Anticancer Res 17:15351539.

Geminder H, Sagi-Assif O, Goldberg L, Meshel T, Rechavi G, Witz IP, Ben-Baruch A. 2001. A possible role for CXCR4 and its ligand, the CXC chemokine stromal cellderived factor-1, in the development of bone marrow metastases in neuroblastoma. J Immunol 167:4747-4757.

Gerard C, Rollins BJ. 2001. Chemokines and disease. Nat Immunol 2:108-115.

Gerritsen ME, Peale FV, Jr., Wu T. 2002. Gene expression profiling in silico: Relative expression of candidate angiogenesis associated genes in renal cell carcinomas. Exp Nephrol 10:114-119.

Grafte-Faure S, Leveque C, Ketata E, Jean P, Vasse M, Soria C, Vannier JP. 2000. Recruitment of primitive peripheral blood cells: Synergism of interleukin 12 with interleukin 6 and stromal cell-derived factor-1. Cytokine 12:1-7.

Guise TA. 2002. How Metastases Home to Bone: The Attraction of Chemokines July 2002 Commentary on: Taichman RS, Cooper C, Keller ET, Pienta KJ, Taichman NS, McCauley LK. Use of the stromal cell-derived factor1/CXCR4 pathway in prostate cancer metastasis to bone. Cancer Res. 2002 Mar 15;62(6):1832-1837. Internet Communication.

Gupta SK, Pillarisetti K. 1999. Cutting edge: CXCR4-Lo: Molecular cloning and functional expression of a novel human CXCR4 splice variant. J Immunol 163:2368-2372.

Hamada T, Mohle R, Hesselgesser J, Hoxie J, Nachman RL, Moore MA, Rafii S. 1998. Transendothelial migration of megakaryocytes in response to stromal cell-derived factor 1 (SDF-1) enhances platelet formation. J Exp Med 188:539-548.

Hodohara K, Fujii N, Yamamoto N, Kaushansky K. 2000. Stromal cell-derived factor-1 (SDF-1) acts together with thrombopoietin to enhance the development of megakaryocytic progenitor cells (CFU-MK). Blood 95:769-775.

Jacobs SC. 1983. Spread of prostatic cancer to bone. Urology 21:337-344.

Kim CH, Broxmeyer HE. 1999. SLC/exodus2/6Ckine/TCA4 induces chemotaxis of hematopoietic progenitor cells: Differential activity of ligands of CCR7, CXCR3, or 
CXCR4 in chemotaxis vs. suppression of progenitor proliferation. J Leukoc Biol 66:455-461.

Korenchuk S, Lehr JE, McLean L, Lee YG, Whitney S, Vessella R, Lin DL, Pienta KJ. 2001. VCaP, a cell-based model system of human prostate cancer. In Vivo 15:163168.

Koshiba T, Hosotani R, Miyamoto Y, Ida J, Tsuji S, Nakajima S, Kawaguchi M, Kobayashi H, Doi R, Hori T, Fujii N, Imamura M. 2000. Expression of stromal cellderived factor 1 and CXCR4 ligand-receptor system in pancreatic cancer: A possible role for tumor progression. Clin Cancer Res 6:3530-3535.

Koutsilieris M. 1993. Osteoblastic metastasis in advanced prostate cancer. Anticancer Res 13:443-449.

Lazarini F, Casanova P, Tham TN, De Clercq E, ArenzanaSeisdedos F, Baleux F, Dubois-Dalcq M. 2000. Differential signalling of the chemokine receptor CXCR4 by stromal cell-derived factor 1 and the HIV glycoprotein in rat neurons and astrocytes. Eur J Neurosci 12:117-125.

Libura JD. 2002. CXCR4-SDF-1 signaling is active in rhabdomyosarcoma cells and regulates locomotion, chemotaxis, and adhesion. Blood 100:2597-2606.

McGrath KE, Koniski AD, Maltby KM, McGann JK, Palis J. 1999. Embryonic expression and function of the chemokine SDF-1 and its receptor, CXCR4. Dev Biol (Orlando) 213:442-456

Mitra P, Shibuta K, Mathai J, Shimoda K, Banner BF, Mori M, Barnard GF. 1999. CXCR4 mRNA expression in colon, esophageal, and gastric cancers and hepatitis $\mathrm{C}$ infected liver. Int J Oncol 14:917-925.

Moore MA. 2001. The role of chemoattraction in cancer metastases. Bioessays 23:674-676.

Muller CA, Homey B, Sato H, Ge N, Catron D, Buchanan M, McClanahan T, Murphy E, Yuan W, Wagners S, Barrera J, Mohar A, Verastegui E, Zlotnik A. 2001. Involvement of chemokine receptors in breast cancer metastasis. Nature 410:50-56.

Nagasawa T, Hirota S, Tachibana K, Takakura N, Nishikawa Y, Kitamura N, Yoshida H, Kikutani T. 1996. Defects of B-cell lymphopoiesis and bone-marrow myelopoiesis in mice lacking the CXC chemokine PBSF/SDF-1. Nature 382:635-638.

Parker SL, Tong T, Bolden S, Wingo PA. 1996. Cancer statistics. CA Cancer J Clin 46:5-27.

Peled A, Petit I, Kollet O, Magid M, Ponomaryov T, Byk A, Nagler H, Ben-Hur A, Many L, Shultz O, Lider R, Alon D, Zipori T. 1999. Dependence of human stem cell engraftment and repopulation of NOD/SCID mice on CXCR4. Science 283:845-848.

Perrone EE, Theoharis C, Mucci NR, Hayasaka S, Taylor JM, Cooney KA, Rubin MA. 2000. Tissue microarray assessment of prostate cancer tumor proliferation in African-American and white men. J Natl Cancer Inst 92:937-939.

Pienta KJ, Esper PS. 1993. Risk factors for prostate cancer. Ann Intern Med 118:793-803.

Putzi MJ, De Marzo AM. 2000. Morphologic transitions between proliferative inflammatory atrophy and highgrade prostatic intraepithelial neoplasia. Urology 56: 828-832.

Rempel SA, Dudas S, Ge S, Gutierrez JA. 2000. Identification and localization of the cytokine SDF1 and its receptor,
CXC chemokine receptor 4, to regions of necrosis and angiogenesis in human glioblastoma. Clin Cancer Res 6:102-111.

Robey PG, Termine JD. 1985. Human bone cells in vitro. Calcif Tissue Int 37:453-460.

Robledo MM, Bartolome RA, Longo N, Rodriguez-Frade JM, Mellado M, Longo I, van MG, Sanchez-Mateos P, Teixido J. 2001. Expression of functional chemokine receptors CXCR3 and CXCR4 on human melanoma cells. J Biol Chem 276:45098-45105.

Rossi D, Zlotnik A. 2000. The biology of chemokines and their receptors. Annu Rev Immunol 18:217-242.

Rottman JB. 1999. Key role of chemokines and chemokine receptors in inflammation, immunity, neoplasia, and infectious disease. Vet Pathol 36:357-367.

Rubin MA, Putzi M, Mucci N, Smith DC, Wojno K, Korenchuk S, Pienta KJ. 2000. Rapid ("warm") autopsy study for procurement of metastatic prostate cancer. Clin Cancer Res 6:1038-1045.

Sehgal A, Keener C, Boynton AL, Warrick J, Murphy GP. 1998. CXCR-4, a chemokine receptor, is overexpressed in and required for proliferation of glioblastoma tumor cells. J Surg Oncol 69:99-104.

Shibuta KM. 2002. Regional expression of CXCL12/CXCR4 in liver and hepatocellular carcinoma and cell-cycle variation during in vitro differentiation. Jpn J Cancer Res 93:789-797.

Shibuta K, Begum NA, Mori M, Shimoda K, Akiyoshi T, Barnard GF. 1997. Reduced expression of the CXC chemokine hIRH/SDF-1alpha mRNA in hepatoma and digestive tract cancer. Int J Cancer 73:656-662.

Taichman RS, Emerson SG. 1994. Human osteoblasts support hematopoiesis through the production of granulocyte colony-stimulating factor. J Exp Med 179:16771682.

Taichman RS, Emerson SG. 1998. The role of osteoblasts in the hematopoietic microenvironment. Stem Cell 16:715

Taichman RS, Cooper C, Keller ET, Pienta KJ, Taichman N, McCauley LK. 2002. Use of the stromal cell-derived factor-1/CXCR4 pathway in prostate cancer metastasis to bone. Cancer Res 62:1832-1837.

Wang JF, Liu ZY, Groopman JE. 1998. The alpha-chemokine receptor CXCR4 is expressed on the megakaryocytic lineage from progenitor to platelets and modulates migration and adhesion. Blood 92:756-764.

Wegner SA, Ehrenberg PK, Chang G, Dayhoff DE, Sleeker al, Michael NL. 1998. Genomic organization and functional characterization of the chemokine receptor CXCR4, a major entry co-receptor for human immunodeficiency virus type 1. J Biol Chem 273:4754-4760.

Weingartner K, Ben-Sasson SA, Stewart R, Richie JP, Riedmiller H, Folkman J. 1998. Endothelial cell proliferation activity in benign prostatic hyperplasia and prostate cancer: An in vitro model for assessment. J Urol 159:465-470.

Zeelenberg IS, Ruuls-Van Stalle L, Roos E. 2001. Retention of CXCR4 in the endoplasmic reticulum blocks dissemination of a T cell hybridoma. J Clin Invest 108:269-277.

Zhou Y, Larsen PH, Hao C, Yong VW. 2002. CXCR4 is a major chemokine receptor on glioma cells and mediates their survival. J Biol Chem M206:200-222. 